\title{
Economic Evaluation of First-Line Pertuzumab Therapy in Patients with HER2-Positive Metastatic Breast Cancer in Japan
}

\author{
Kensuke Moriwaki ${ }^{1,2} \odot$ Saki Uechi ${ }^{2} \cdot$ Takaaki Fujiwara $^{2} \cdot$ Yu Hagino $^{2} \cdot$ Kojiro Shimozuma $^{1,3}$
}

Accepted: 27 December 2020 / Published online: 23 January 2021

(c) The Author(s) 2021

\begin{abstract}
Objective The purpose of this analysis was to evaluate the cost effectiveness of the combination of pertuzumab, trastuzumab, and docetaxel (PTD) for the treatment of patients with human epidermal growth factor receptor-2 (HER2)-positive breast cancer in Japan.

Methods A partitioned survival analysis model was developed to predict costs and quality-adjusted life-years (QALYs) in a PTD arm and a trastuzumab plus docetaxel (TD) arm. Direct medical costs were considered from the perspective of the Japanese healthcare system. The time horizon of the model was set to 20 years. Data on overall survival and progressionfree survival were derived from the CLEOPATRA trial. Cost parameters were estimated using a real-world claims database. Utilities were derived from published sources outside Japan. The incremental cost-effectiveness ratio (ICER) of PTD therapy compared with TD therapy was estimated. Sensitivity analysis was conducted to assess the uncertainty in parameter settings. Results Compared with TD therapy, PTD therapy incurred an additional cost of \$US174,479 and conferred an additional 0.949 QALYs. This resulted in an ICER of \$US183,901 per QALY gained. Utility weights for progression-free survival and progressed disease had a relatively large impact on the base-case result, but the ICERs remained higher than \$US75,000 per QALY over the full range of model parameters. Based on a probabilistic sensitivity analysis, the probability that PTD is cost effective was estimated to be $3.3 \%$.

Conclusions Applying a willingness-to-pay threshold of \$US75,000 per QALY, PTD therapy as first-line therapy would not be cost effective. Further research is required on utilities and clinical benefits for Japanese patients with breast cancer.
\end{abstract}

Supplementary Information The online version contains supplementary material available at https://doi.org/10.1007/s4166 9-020-00254-3.

Kensuke Moriwaki

moriken820318@gmail.com

1 Comprehensive Unit for Health Economic Evidence Review and Decision Support (CHEERS), Research Organization of Science and Technology, Ritsumeikan University, \#209, Research Park Bid. No. 2, 134, Minami-machi, Chudoji, Simogyo-ku, Kyoto 600-8813, Japan

2 Laboratory of Medical Statistics, Kobe Pharmaceutical University, 4-19-1, Motoyamakita-machi, Higashinada-ku, Hyogo 658-8558, Japan

3 Division of Health Service Research, Department of Biomedical Science, College of Life Sciences, Ritsumeikan University, 1-1-1, Noji-higashi, Kusatsu, Shiga 525-8577, Japan

\section{Introduction}

In recent years, the incidence of breast cancer has been increasing in Japan. The age-adjusted incidence was 57.3 cases per 100,000 people in 2005 and 95.4 cases per 100,000 people in 2015, an increase of 1.67 times in 10 years [1]. According to estimated Japanese national medical care expenditure in 2017, the annual total medical costs for malignant neoplasms were \$US43.766 billion (assuming \$US1= 100 Japanese yen [JPY]), of which breast cancerrelated costs were \$US3.495 billion, accounting for 7.99\% of total cancer costs [2]. Breast cancer-related expenditures have increased $38 \%$ over the last decade, and the socioeconomic burden of breast cancer tends to increase year by year [2].

Human epidermal growth factor receptor 2 (HER2)positive breast cancer, which accounts for approximately $20 \%$ of all breast cancer, has a higher malignancy rate and a poorer prognosis than HER2-negative breast cancers [3]. 


\section{Key Points for Decision Makers}

Although current guidelines recommend the combination of pertuzumab, trastuzumab, and docetaxel (PTD) as a first-line therapy for patients with human epidermal growth factor receptor-2 (HER2)-positive breast cancer, the cost effectiveness of PTD in Japan remains controversial.

Our results indicate that PTD therapy would not be cost effective from the Japanese public healthcare payer's perspective and were robust except for some settings related to patient quality of life.

In April 2019, Japan formally implemented a cost-effectiveness evaluation system to adjust the prices of health technologies.

If the price adjustment rule is applied to our results, the incremental cost-effectiveness ratio of pertuzumab (\$US183,901 per quality-adjusted life-year [QALY]) exceeds \$US150,000 per QALY; therefore, the premium price for pertuzumab would be reduced by $90 \%$.

The current clinical guidelines recommend combination therapy with pertuzumab plus trastuzumab plus docetaxel (PTD) as a first-line therapy for patients with HER2-positive metastatic or recurrent breast cancer [4]. Pertuzumab is a molecularly targeted drug approved in Japan in 2013, and its additional clinical benefit over trastuzumab plus docetaxel (TD) alone was confirmed in a randomized controlled trial (CLEOPATRA) [5].

While pertuzumab is an efficacious drug, it is relatively expensive, and there is interest in determining its cost effectiveness [4]. Several cost-effectiveness analyses of pertuzumab as a first-line treatment for HER2-positive metastatic breast cancer outside Japan have previously estimated the incremental cost-effectiveness ratio (ICER) of PTD compared with TD [6-9]. These studies indicated that PTD combination therapy appears not to be more cost effective than control treatment, applying a willingness-to-pay (WTP) threshold of \$US50,000-100,000 per quality-adjusted life-year (QALY) [6-9]. However, no studies to date have examined the cost effectiveness of pertuzumab as a first-line therapy for HER2-positive metastatic breast cancer from the Japanese healthcare payer's perspective.

Evidence from the previous studies may not be applicable to healthcare decision making in Japan given the differences in healthcare systems, including drug prices, treatment fees, and socially acceptable thresholds for the ICER. Since 2019, clinical and economic evidence has been used to adjust the prices of health technologies in Japan [10]. As such, economic evaluations from the Japanese public healthcare payer's perspective will be increasingly important, especially in the oncology field, where treatments are becoming more expensive. Therefore, we conducted a model-based economic evaluation to assess the cost effectiveness of PTD combination therapy in patients with HER2-positive metastatic or recurrent breast cancer, aiming to support healthcare decision making in Japan.

\section{Methods}

\subsection{Model-Based Cost-Effectiveness Analysis}

A model-based, cost-effectiveness analysis was conducted to evaluate the cost effectiveness of PTD combination therapy from the perspective of Japan's health insurance payer. We targeted Japanese women with HER2-positive metastatic or recurrent breast cancer without a treatment history as a hypothetical cohort. The current clinical guideline recommends PTD as a first-line therapy for patients with HER2positive metastatic or recurrent breast cancer [4]. Trastuzumab plus chemotherapy or trastuzumab emtansine is also recommended as the next-best treatment [4]. In the current study, we selected TD as a comparator in terms of the availability of evidence for the additional benefit of pertuzumab. In this study, we assumed that the characteristics of Japanese patients with HER2-positive breast cancer were similar to those of the patient population of CLEOPATRA [5].

We developed a partitioned survival analysis (PartSA) model to predict long-term costs and QALYs associated with each therapy [11]. In the PartSA model, the prognosis of patients with breast cancer was modeled in three states: "survival in the progression-free state," "survival after progression," and "death" [11]. Utility weights and costs per cycle were set for each health condition. For cost-effectiveness analysis based on PartSA, we applied parametric functions (the Weibull function, lognormal function, etc.) to the Kaplan-Meier curves of progression-free survival (PFS) and overall survival (OS) obtained in the CLEOPATRA trial [5]. Next, using the parametric functions fitted to PFS and OS, we calculated the change over time of the proportion of patients existing in each state. By multiplying the estimated patient proportion with the cost per cycle and utility value of each condition, the expected long-term costs and QALYs were calculated.

Based on the estimated expected costs and expected QALYs, the ICER was estimated as an indicator of cost effectiveness using the following formula: ICER $=($ Cost ${ }_{\text {PTD }}-$ Cost $\left._{\mathrm{TD}}\right) /\left(\mathrm{QALY}_{\mathrm{PTD}}-\mathrm{QALY}_{\mathrm{TD}}\right)$. A WTP threshold of \$US75,000 (JPY7.5 million, assuming \$US1 = JPY100) per QALY gained was used as the acceptable level for the ICER of anticancer drugs [12]. The time horizon of the analysis was set to 20 years. Based on cost-effectiveness 
assessment guidelines in Japan, a discount rate of $2 \%$ per annum was applied to long-term costs and the QALYs [13]. The life-years were also discounted. The cycle length of the model was defined as 1 month. Half-cycle correction was used in the simulation process. The model was developed and analyzed using TreeAge Pro 2019 (TreeAge Software LLC. Williamstown, MA, USA).

\subsection{Curve Fitting}

To estimate the PFS and OS of each treatment group required for simulation using PartSA, a parametric function fitting (curve fitting) to the Kaplan-Meier curves of the clinical trial was performed. Curve fitting requires patient-level data of survival time collected in clinical trials, but generally these are not published or provided, so alternative methods need to be considered. In this study, we created a patientlevel time-to-event data set using the methods proposed by Guyot et al. [14]: (1) we extracted plot information of time and cumulative survival probability from the image data of the Kaplan-Meier curves; (2) based on the plot information and the number-at-risk data reported in the clinical trial, we created the patient-level time-to-event data set.

WebPlotDigitizer (version 4.3) was used to extract plot information. We used R (v. 3.6.3) to create the patient-level data set. We used the image data of the Kaplan-Meier curves on PFS and OS in the CLEOPATRA trial to create a patientlevel time-to-event data set $[5,14]$. In the curve fitting, the following parametric functions were used: (1) exponential function, (2) Weibull function, (3) lognormal function, (4) log-logistic function, (5) Gompertz function, and (6) generalized gamma function. Curve fitting was performed using Stata 16 (Stata Corp LLC., College Station, TX, USA). The optimal function for base-case analysis was determined based on visual inspection and statistics such as Akaike information criteria (AIC) and Bayesian information criteria (BIC). The AIC and BIC for the fitted curves are shown in Table 1 in the electronic supplementary material (ESM). We selected the Weibull function and lognormal function for the OS and PFS curves, respectively. Additionally, we assumed that the proportional hazards assumptions between treatment groups were met, and we developed Weibull and lognormal models including the covariate of the treatment effect of PTD (Table 1).

\subsection{Costs}

We considered only direct medical costs from the perspective of the Japanese healthcare system. Table 1 summarizes the input values for the cost parameters. All costs were calculated in JPY and converted to \$US with a currency exchange rate of $\$$ US $1=J P Y 100$ (as in 2020). In this model, the following cost parameters were set: (1) monthly treatment costs before disease progression in each group, (2) monthly other medical costs before disease progression in each group, (3) monthly medical costs after disease progression, and (4) end-of-life medical costs (per case).

The above cost parameters were estimated using the claims database provided by Japan Medical Data Center Co., Ltd. (JMDC). The JMDC claims database is the largest real-world database for commercial use in Japan, and it collects subscriber registry, receipts (hospitalization, outpatient, and dispensing receipts), and medical examination data received from insurers. In this database, a unique identifier is assigned to each subscriber so that tracking is possible even if a transfer takes place or multiple facility visits occur. However, elderly people (aged $>75$ years) are not included. In this research, from January 2005 to January 2017, data for 17,745 subjects that included the following International Classification of Diseases, Tenth Revision codes related to breast cancer were extracted and analyzed: C50.0, C50.1, C50.2, C50.3, C50.4, C50.6, C50.8, C50.9, C78.0, C79.2, C79.5, C79.8, D05.9, and T88.9. Although the medical fee schedule in Japan is revised once every 2 years, the cost parameters were estimated based on the unit price at the time the medical resources were consumed.

The average medication expenses for 1 month for the use of PTD therapy and TD therapy was estimated as the drug costs of the progression-free state in each group. Similarly, the average medical expenses other than drug treatment expenses for 1 month for the use of PTD therapy and TD therapy was estimated as the other medical costs of the progression-free state in each group. The average monthly medical expenses since the last month that PTD therapy was used was estimated as the medical costs after disease progression. We assumed that the monthly medical costs after disease progression were the same in both treatment arms because the treatment pattern received by patients who discontinued PTD or TD was similar in the CLEOPATRA trial [5]. The total medical expenses for 3 months before death were defined as the terminal care cost and estimated as endof-life medical costs per case. Additionally, we assumed that the consumption of medical resources in the terminal phase was the same in both treatment arms.

\subsection{Utility Weights}

In this analysis, the utility weights before the disease progression state and after the disease progression state in each treatment group were set as shown in Table 1. The baseline utility weights of the progression-free state in the PTD and TD arms were derived from a report valuing the health status of patients with breast cancer using the standard gamble method (SG) in the general population in UK $[15,16]$. In addition, disutility due to adverse events (AEs) was considered in the progression-free state [15-17]. Disabilities 
Table 1 Survival curve settings and parameter inputs

\begin{tabular}{|c|c|c|c|c|c|c|}
\hline \multirow{2}{*}{$\begin{array}{l}\text { Settings and inputs } \\
\text { Survival function }\end{array}$} & \multirow[t]{2}{*}{ Estimate } & \multirow[t]{2}{*}{ SE } & \multicolumn{2}{|c|}{ Plausible range } & \multirow[t]{2}{*}{ Distribution } & \multirow[t]{2}{*}{ References } \\
\hline & & & & & & \\
\hline \multicolumn{7}{|l|}{ Parameter for Weibull function for OS } \\
\hline Covariate of efficacy of PTD vs. TD & 0.6753 & 0.0700 & 0.5512 & 0.8274 & Gamma & {$[5,14]$} \\
\hline Ramada & 0.0059 & 0.0013 & 0.0038 & 0.0092 & Gamma & {$[5,14]$} \\
\hline Kappa & 1.2654 & 0.0580 & 1.1568 & 1.3843 & Gamma & {$[5,14]$} \\
\hline \multicolumn{7}{|l|}{ Parameter for lognormal function for PFS } \\
\hline Covariate of efficacy of PTD vs. TD & 0.4174 & 0.0851 & 0.2507 & 0.5841 & Gamma & {$[5,14]$} \\
\hline $\mathrm{Mu}$ & 2.6037 & 0.0597 & 2.4866 & 2.7208 & Gamma & {$[5,14]$} \\
\hline Sigma & 1.1510 & 0.0348 & 1.0847 & 1.2214 & Gamma & {$[5,14]$} \\
\hline \multicolumn{7}{|l|}{ Cost parameter } \\
\hline \multicolumn{7}{|c|}{ Drug treatment cost before disease progression } \\
\hline P (\$US per month) & 3263 & - & 3247 & 3340 & - & JMDC \\
\hline TD (\$US per month) & 2529 & 62 & 2409 & 2650 & Gamma & JMDC \\
\hline \multicolumn{7}{|c|}{ Other medical cost before disease progression } \\
\hline PTD arm (\$US per month) & 1007 & 47 & 915 & 1100 & Gamma & JMDC \\
\hline TD arm (\$US per month) & 1245 & 88 & 1,073 & 1418 & Gamma & JMDC \\
\hline \multicolumn{7}{|l|}{ Medical cost after disease progression ${ }^{\mathrm{a}}$} \\
\hline PTD arm (\$US per month) & 5404 & 244 & 4925 & 5883 & Gamma & JMDC \\
\hline TD arm (\$US per month) & 5404 & 244 & 4925 & 5883 & Gamma & JMDC \\
\hline \multicolumn{7}{|l|}{ End-of-life medical cost (\$US per case $)^{\mathrm{a}}$} \\
\hline PTD arm (\$US per month) & 14,340 & 178 & 13,992 & 14,689 & Gamma & JMDC \\
\hline TD arm (\$US per month) & 14,340 & 178 & 13,992 & 14,689 & Gamma & JMDC \\
\hline \multicolumn{7}{|l|}{ Utility parameter } \\
\hline \multicolumn{7}{|l|}{ Baseline utility before disease progression } \\
\hline PTD arm & 0.786 & 0.163 & 0.485 & 0.935 & Beta & {$[15,16]$} \\
\hline TD arm & 0.786 & 0.163 & 0.485 & 0.935 & Beta & {$[15,16]$} \\
\hline \multicolumn{7}{|l|}{ Disutility due to AE event } \\
\hline PTD arm & 0.056 & - & 0.016 & 0.098 & Triangle & {$[15-17]$} \\
\hline TD arm & 0.040 & - & 0.011 & 0.058 & Triangle & {$[15-17]$} \\
\hline \multicolumn{7}{|l|}{ Cycle probability of $\mathrm{AE}$ event } \\
\hline PTD arm (per month) & 0.0130 & 0.0033 & 0.0043 & 0.0173 & Beta & {$[15,18]$} \\
\hline TD arm (per month) & 0.0087 & 0.0022 & 0.0043 & 0.0130 & Beta & {$[15,18]$} \\
\hline \multicolumn{7}{|l|}{ Utility after disease progression } \\
\hline PTD arm & 0.538 & 0.163 & 0.196 & 0.848 & Beta & {$[15,16]$} \\
\hline TD arm & 0.538 & 0.163 & 0.196 & 0.848 & Beta & {$[15,16]$} \\
\hline Discount rate $(\%)$ & 2 & - & 0 & 4 & - & [13] \\
\hline
\end{tabular}

$A E$ adverse event, $J M D C$ Japan Medical Data Center Co., Ltd. database, $O S$ overall survival, $P$ pertuzumab, $P F S$ progression-free survival, $P T D$ combination therapy of pertuzumab, trastuzumab, and docetaxel, $S E$ standard error, $T D$ combination therapy of trastuzumab and docetaxel

${ }^{a}$ Assumed the same in both arms.

associated with AEs were not necessarily limited to breast cancer and were indirectly estimated from data based on the SG and assumptions [15, 17]. The monthly probability of AE in the PTD and TD arms was calculated based on the values reported in a previous study and in the CLEOPATRA trial $[15,18]$. The utility weights after disease progression in the PTD and TD arms were assumed to be the same $[15,16]$.

\subsection{Sensitivity Analysis}

In this study, one-way sensitivity analysis was performed on the parameters set in the model. Here, $95 \%$ confidence intervals (CIs) were used for the plausible ranges of the survival function and cost parameters (Table 1). Regarding the plausible range of drug costs for pertuzumab, we calculated and set the worst-case and best-case price based on the Japanese price adjustment system. Under the current Japanese 
health technology assessment (HTA) system, the premium drug price is adjusted according to the cost-effectiveness level of the drug. In the best case, the premium increases by $50 \%$, whereas in the worst case, the premium decreases by $90 \%$ [10]. Pertuzumab was given a $5 \%$ premium increase to the base price at approval [19]. Therefore, the worst case and best case drug cost for pertuzumab was as follows: worstcase cost $=\$$ US3107.4 (base cost) +155.4 (premium) $\times 0.1$ $(90 \%$ decreased $)=\$$ US3247.2 ; best-case cost $=\$$ US3107.4 $($ base cost $)+155.4($ premium $) \times 1.5(50 \%$ increased $)=$ $\$$ US3340.4 (Table 1). For the utility parameters, we used the values reported in the previous study to set their plausible ranges (Table 1). The results of the sensitivity analyses are summarized as a tornado diagram.

In this study, scenario sensitivity analyses on the selection of parametric functions for OS and PFS were conducted. We also performed scenario analyses applying the results of subgroup analysis in the CLEOPATRA trial [5]. The subgroup analysis showed that the hazard ratios of OS and PFS in the Asian population (261 patients) were 0.82 (95\% CI $0.58-1.17$ ) and 0.77 (95\% CI 0.58-1.03), respectively [5]. In addition, the subgroup analysis in the Japanese population (53 patients) indicated that the hazard ratios of OS and PFS were 1.17 (95\% CI 0.36-3.88) and 1.92 (95\% CI 0.91-4.04), respectively [20]. These hazard ratios were applied to the OS and PFS curves in the TD arm to predict the OS and PFS in the PTD arm.

Probabilistic sensitivity analyses were performed using 1000 iterations of a second-order Monte Carlo simulation to examine the influence of parameter uncertainty on the cost effectiveness. Probabilistic distributions of the parameters used in the probabilistic sensitivity analyses are shown in Table 1. We applied a relevant distribution for each variable in the model if data on variability were available. In each of the 1000 simulations, the value for each model input was randomly selected from its distribution. Based on the results of the second-order Monte Carlo simulation, we constructed cost-effectiveness acceptability curves and estimated the proportion of iterations in which PTD therapy would be preferred in terms of cost effectiveness, assuming a WTP threshold of \$US75,000 per QALY gained.

\section{Results}

\subsection{Base-Case Analysis}

Changes in the proportion of patients in progression-free and progressed disease (PD) states for each group were estimated as shown in Fig. 1a and b. The results of the base-case analysis are shown in Table 2. The average survival time in the progression-free state was 2.954 years in the PTD arm and 2.039 years in the TD arm. Additionally, the average survival time in the PD state was 2.672 years in the PTD arm and 2.243 years in the TD arm. Furthermore, the QALYs of the PTD and TD groups were 3.725 and 2.776, respectively. Pertuzumab conferred 0.949 additional QALYs. Meanwhile, the total costs in the PTD and TD groups were \$US423,509 and 249,029, respectively. The incremental cost of using pertuzumab was $\$$ US 174,479. From these results, the ICER of PTD therapy compared with TD therapy was estimated to be \$US183,901 per QALY gained.

\subsection{Sensitivity Analysis}

The results of the one-way sensitivity analysis are shown in a tornado diagram (Fig. 2). Factors that greatly affected the cost effectiveness of pertuzumab were the utility values of the progression-free and PD states. The parameter that most strongly affected the ICER of pertuzumab was the utility value of the PD state in PTD therapy. The best-case ICER was estimated at \$US98,189 per QALY, and the worst-case ICER was estimated at $\$$ US4,974,429 per QALY. Compared with that of the utility settings, the effect of the other parameters on the ICER was limited.

The results of the scenario analyses on the selection of parametric functions for OS and PFS are summarized in Table 2 and Fig. 1 in the ESM. The best-case ICER was estimated at \$US168,084 per QALY in the exponential curve setting, and the worst-case ICER was \$US203,524 per QALY in the Gompertz curve settings. The ICERs remained higher than \$US75,000 per QALY over the full range of model parameters and in all curve settings. The results of the scenario analyses applying the treatment effect of subgroup in the CLEOPATRA trial are summarized in Tables 3 and 4 in the ESM. Applying the treatment effect in the Asian subgroup, the ICER of PTD therapy was estimated to be \$US460,726 per QALY gained. Assuming the treatment effect in the Japanese subgroup, PTD therapy was dominated by TD therapy.

The results of the probabilistic sensitivity analyses are summarized in Fig. 3a. Cost-effectiveness acceptability curves constructed on the basis of the scatter plot are shown in Fig. 3b. Using a WTP threshold of \$US75,000 per QALY gained, the probability that PTD therapy was more cost effective than TD therapy was estimated to be $3.3 \%$.

\section{Discussions}

Although the current Japanese guidelines recommend PTD as a first-line therapy for patients with HER2-positive metastatic or recurrent breast cancer, the cost effectiveness of PTD in a real-world setting in Japan remains controversial [4]. In this economic evaluation, we estimated the cost effectiveness of adding pertuzumab to TD in treating 
Fig. 1 Results of survival curve fitting and cohort tracing. a Pertuzumab, trastuzumab, and docetaxel arm. b Trastuzumab and docetaxel arm. $O S$ overall survival, $P F S$ progression-free survival, $P T D$ combination therapy of pertuzumab, trastuzumab, and docetaxel, TD combination therapy of trastuzumab and docetaxel
(A)

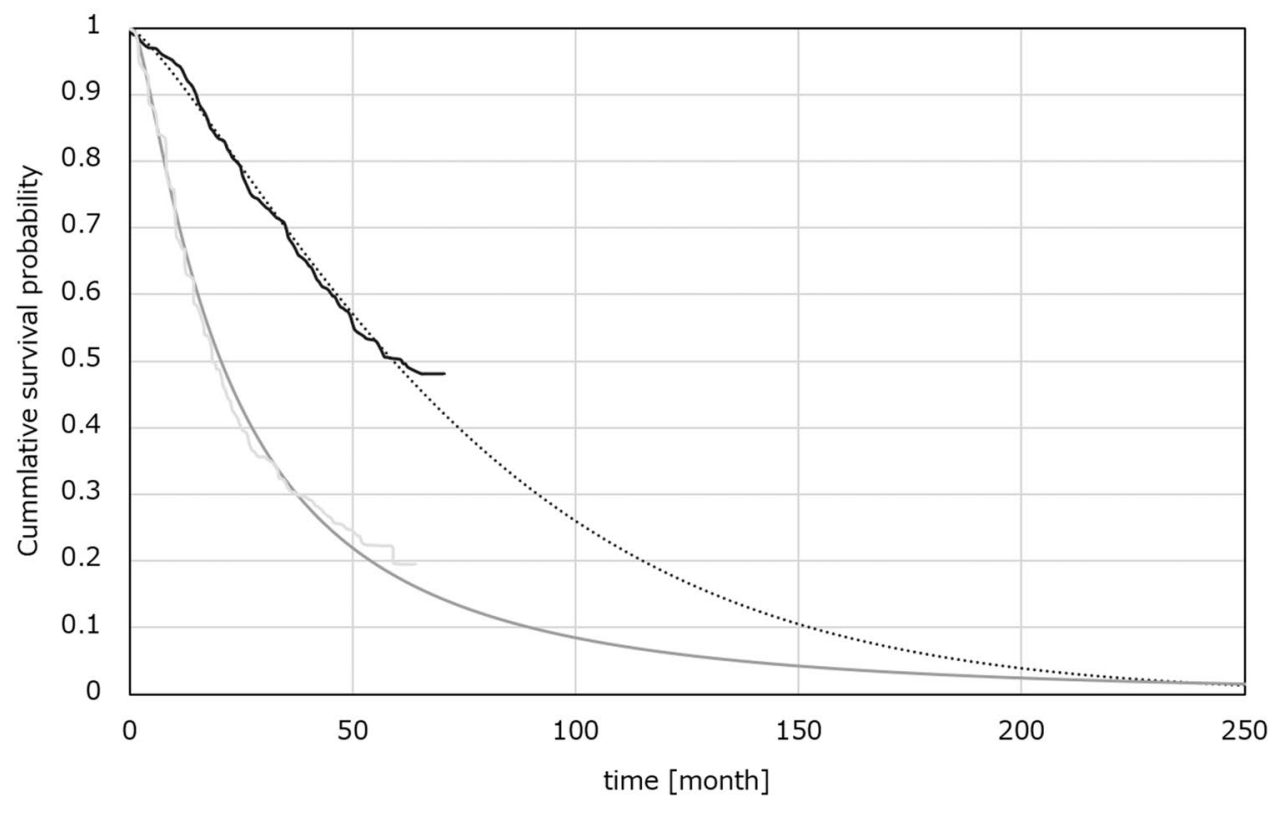

... OS_PTD_weibull_—PFS_PTD_lognormal — OS_PTD — PFS_PTD

(B)

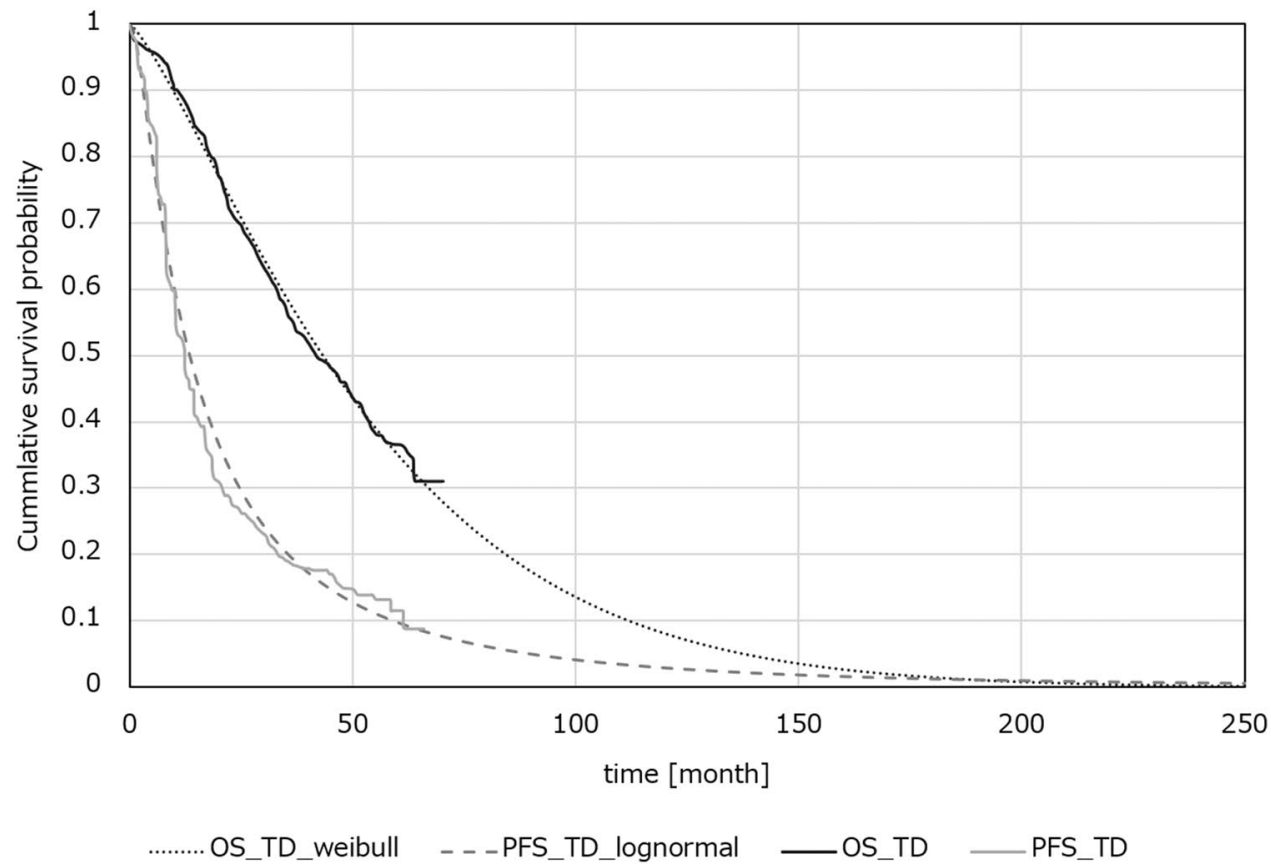

HER2-positive metastatic breast cancer from the Japanese public healthcare payer's perspective. Durkee et al. [6] performed a cost-effectiveness analysis on pertuzumab as a first-line treatment for HER2-positive metastatic breast cancer from the payer's perspective in the USA and estimated the ICER of PTD compared with TD to be \$US472,668 per QALY gained [6]. In addition, Diaby et al. [7] also conducted an economic evaluation of PTD from the payer's perspective in the USA, reporting an ICER of pertuzumab of \$US344,410-398,444 per QALY. Furthermore, Leung et al. [8] estimated the ICER of PTD compared with TD to be \$US593,741 per QALY by using the national claims database in Taiwan. Consistent with previous studies, we found that PTD was unlikely to be cost effective at a WTP 
threshold of \$US75,000 per QALY gained. The ICER of PTD compared with TD tended to be lower than that of previous studies. While the ICER in the Japanese setting was \$US183,901 per QALY gained, the ICER in the other countries ranged from $\$$ US344,410 to 593,741 per QALY [6-8]. There are several possible explanations for this gap, one of which is the difference in the drug price of pertuzumab, which is lower in Japan than in other countries [6, 7]. According to the Japanese drug price standard, the drug price of pertuzumab was calculated to be \$US2318.86 per $420 \mathrm{mg} / 14 \mathrm{ml}$ single vial, which is approximately $60 \%$ of the foreign average price (\$US3853.27 per $420 \mathrm{mg} / 14 \mathrm{ml}$ single vial) [19].

Another factor influencing this discrepancy is the discount rate used for the simulation. In previous studies in the USA, a discount rate of 3-3.5\% was applied, whereas we used a discount rate of $2 \%$ in accordance with the guidelines for cost-effectiveness assessment in Japan $[6,7,13]$. Because of this difference, the incremental effectiveness in our analysis was somewhat higher, and the ICER might be estimated to be relatively low.

In previous studies, a Markov model that takes into account the health state, such as post-second-line treatment and hospice care, was used, whereas a PartSA model was used in the present analysis [6-8]. We considered the PartSA model to be a better approach for a variety of reasons. First, we aimed to avoid the uncertainty that comes from additional assumptions depending on the complex Markov model structure. Second, we needed to simplify the parameter settings, such as risk, efficacy, cost, and utility weight, because of limitations in the data availability in Japan. Third, the structure of the PartSA model had better affinity with the endpoints used in clinical trials for anticancer treatment, such as OS and PFS. In recent years, Guyot et al. [14] proposed a method of constructing pseudo-patient-level survival time data by simulation from the image data of the Kaplan-Meier curves and the number-at risk table. Guyot's method is widely used in economic evaluation and has been suggested to be superior in performance than other methods [21]. Although we did not have access to a patient-level timeto-event dataset in a clinical trial such as CLEOPATRA, this proposed method allowed a cost-effectiveness analysis based on the PartSA model with pseudo-patient-level data. Finally, in recent years, the PartSA model has been widely accepted in the process of HTA for metastatic/recurrent cancer in HTA organizations such as the UK National Institute for Health and Care Excellence [11].

The novelty of this study was in clarifying the cost effectiveness of PTD using a real-world claims database that reflects clinical practice in Japan. The JMDC claims database is the largest epidemiological receipt database for commercial use in Japan. In this database, a unique ID is assigned to each patient, and it is possible to track patients 


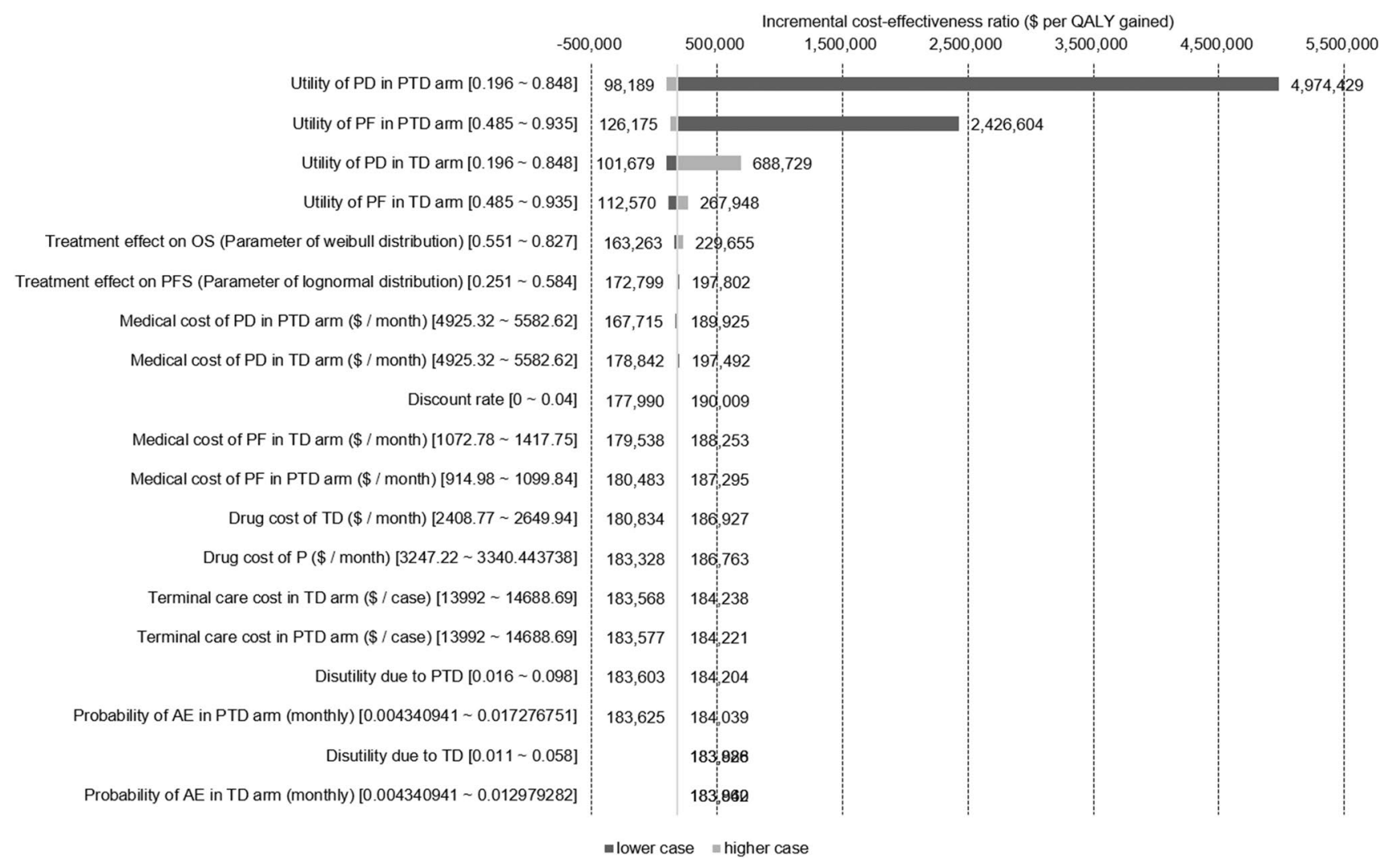

Fig. 2 Results of deterministic sensitivity analyses

even if they are transferred to another hospital or visit multiple facilities. By using this database, we were able to estimate the ICER, taking into account the real-world settings in Japan, including medical fees, drug prices, and medical resource consumption. From the results of the one-way sensitivity analysis, the influence of uncertainty in estimating cost parameters was considered to be limited.

In Japan, a new HTA system that utilizes economic evaluation evidence in adjusting the price of new healthcare technology was started in FY2019 [10, 12]. This system adopts a rule to recalculate the premium in the price of a target technology according to its ICER level. In the process of repricing technologies that require special considerations, such as anticancer drugs, three threshold values for the ICER were set, as follows: \$US75,000, \$US112,500, and \$US150,000 per QALY [12]. If the ICER is less than \$US75,000 per QALY, no price adjustment is made [12]. On the other hand, if the ICER exceeds \$US75,000 per QALY, the premium is recalculated in stages as follows: (1) the premium is reduced by $30 \%$ if the ICER ranges from $\$$ US75,000 to 112,500 per QALY, (2) the premium is reduced by $60 \%$ if the ICER ranges from $\$$ US112,500 to 150,000 per QALY, and (3) the premium is reduced by $90 \%$ if the ICER exceeds \$US150,000 per QALY [12]. Pertuzumab was given a 5\% premium increase to the base price at approval [19]. If this rule is applied to our results, the ICER of pertuzumab (\$US183,901 per QALY) exceeds \$US150,000 per QALY; therefore, the premium will be adjusted to be $0.5 \%(5 \% \times$ $0.1)[12,19]$. Although pertuzumab has not been selected as a target technology, our approach using a PartSA model is useful and applicable, particularly in the evaluation of expensive anticancer drugs, because the estimate of ICER is directly linked to decision making in price adjustments in Japan. Our approach is expected to support policy decisions such as price adjustments.

There are three main limitations to this study. First, we used utility weights derived from previous studies performed in countries other than Japan because of a lack of domestic data. The results of the one-way sensitivity analysis indicated that the plausible range of utility parameters was wide and that the influence on the ICER was relatively large. In particular, the utility weight of the PD state in the PTD arm had the greatest influence, which resulted in the best-case ICER of \$US98,189 per QALY. If the Japanese repricing rule was applied, the decision making in price adjustment might change. Therefore, to improve the accuracy and precision of ICER estimates, it is important to accumulate utility data from patients with breast cancer that reflect Japanese preferences. 
Fig. 3 Results of probabilistic sensitivity analyses. a Scatter plot. b Cost-effectiveness acceptability curve. $Q A L Y$ quality-adjusted life-year, $P T D$ combination therapy of pertuzumab, trastuzumab, and docetaxel, $T D$ combination therapy of trastuzumab and docetaxel
(A)

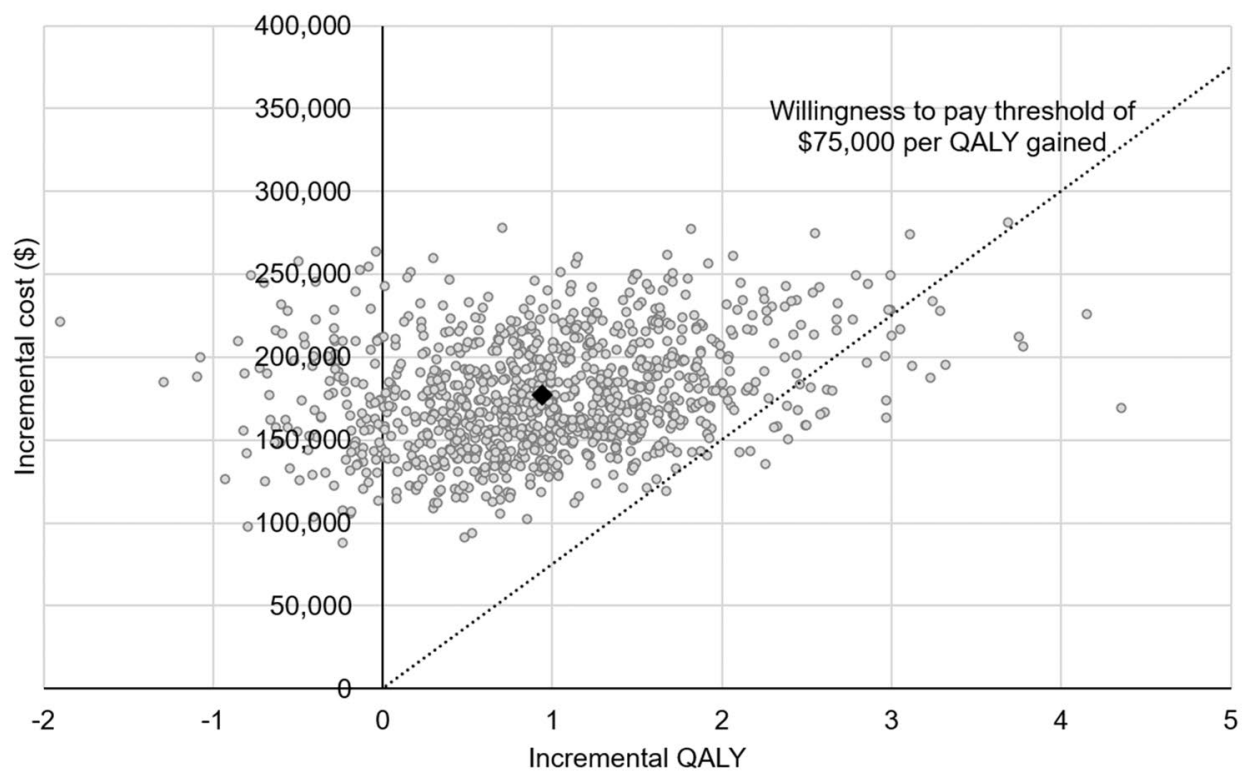

(B)

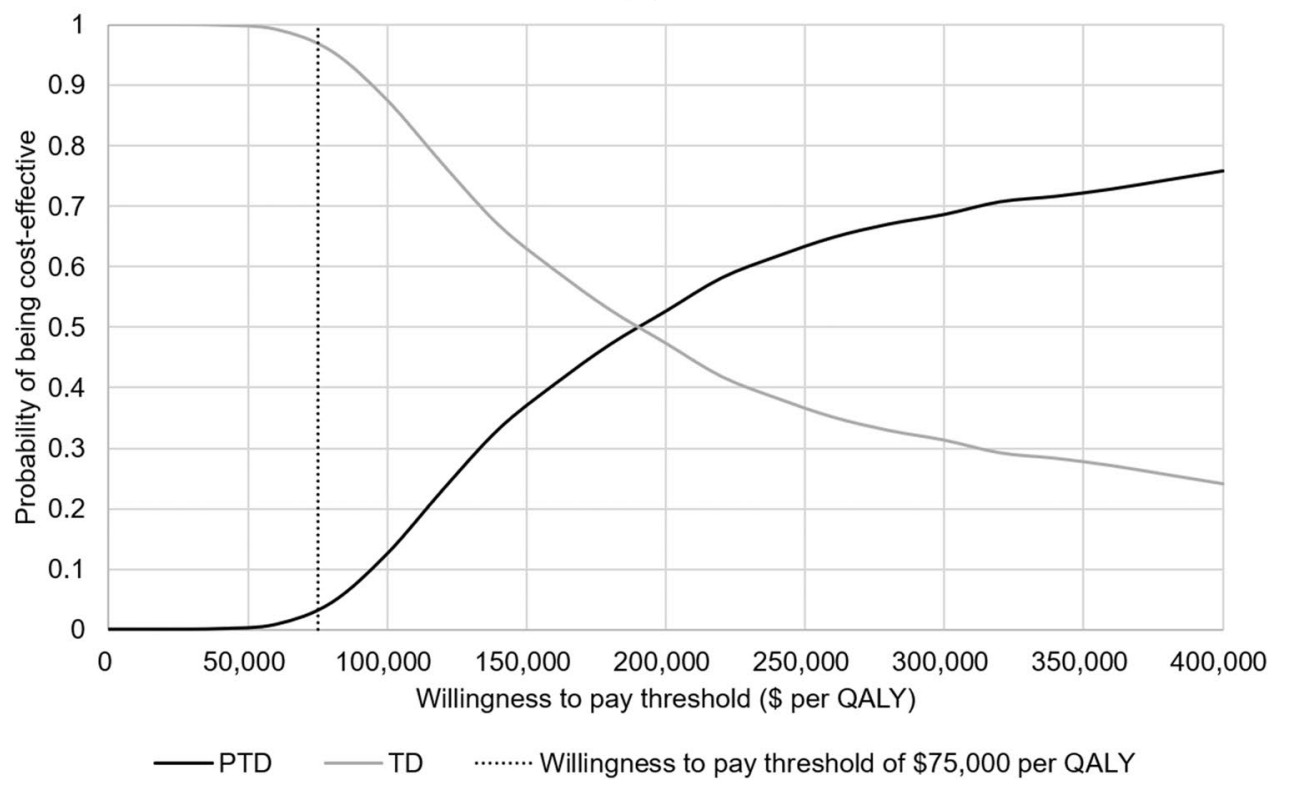

Second, the CLEOPATRA study indicated race-dependent heterogeneity in estimating the efficacy of pertuzumab [5]. Hazard ratios (HRs) for OS and PFS were reported to be 0.67 (95\% CI 0.55-0.82) and 0.68 (95\% CI 0.58-0.80), respectively, among all patients (808 patients) in the CLEOPATRA trial [5]. On the other hand, the subgroup analysis showed that the HRs of OS and PFS in the Asian population (261 patients) were 0.82 (95\% CI 0.58-1.17) and 0.77 (95\% CI 0.58-1.03), respectively [5]. In addition, the subgroup analysis in the Japanese population (53 patients) indicated that the HRs of OS and PFS were 1.17 (95\% CI 0.36-3.88) and 1.92 (95\% CI 0.91-4.04), respectively [20].
These results indicate that the clinical benefit of pertuzumab tends to be smaller among Asian patients than among other populations. Our results from the scenario analyses assuming the treatment effect in the Asian subgroup showed that the ICER of PTD therapy (\$US460,726 per QALY) was higher than the base-case result (\$US183,901 per QALY). Also, the scenario analyses indicated that PTD therapy was dominated by TD therapy applying the treatment effect in the Japanese subgroup. Although the subgroup analysis should be interpreted with caution because of the small sample size, imbalance of background factors between treatment groups, and lack of statistical power, our analysis might have 
overestimated the incremental effect and underestimated the ICER.

Third, there were specific limitations in cost estimation based on claims data such as comparability between PTD and TD group, the separability of unrelated medical costs, and data availability in the older population. Although sensitivity analyses showed that cost parameters had a limited impact on the base-case result, these limitations might systematically bias the ICER estimate.

\section{Conclusions}

For a WTP threshold of \$US75,000 per QALY, PTD as first-line therapy would not be cost effective. Further study is required on utility and clinical benefits in the Japanese population.

\section{Declarations}

Author contributions Study design: KM and SU Data collection: KM, SU, TF, and YH. Data analysis: KM, SU, TF, and YH. Data interpretation: KM, SU, and KS. Manuscript drafting: KM, SU, and KS. Manuscript content revision: KM, SU, and KS. Final manuscript version approval: KM, SU, TF, YH, and KS. KM takes responsibility for the integrity of the data analysis.

Funding This work was supported by Grants-in-Aid for Scientific Research (KAKENHI) grant number 17H04099 from the Ministry of Education, Culture, Sports, Science and Technology (MEXT)/Japan Society for the Promotion of Science.

Conflicts of interest Kensuke Moriwaki, Saki Uechi, Takaaki Fujiwara, Yu Hagino, Kojiro Shimozuma have no conflicts of interest that are directly relevant to the content of this article.

Ethical approval This article does not contain any studies with human participants or animals performed by any of the authors.

Consent to participate Not applicable.

Consent for publication Not applicable.

Code availability Not applicable.

Data availability Some of the data generated during and/or analyzed during the current study are not publicly available but are available from the corresponding author on request.

Open Access This article is licensed under a Creative Commons Attribution-NonCommercial 4.0 International License, which permits any non-commercial use, sharing, adaptation, distribution and reproduction in any medium or format, as long as you give appropriate credit to the original author(s) and the source, provide a link to the Creative Commons licence, and indicate if changes were made. The images or other third party material in this article are included in the article's Creative Commons licence, unless indicated otherwise in a credit line to the material. If material is not included in the article's Creative Commons licence and your intended use is not permitted by statutory regulation or exceeds the permitted use, you will need to obtain permission directly from the copyright holder. To view a copy of this licence, visit http://creativecommons.org/licenses/by-nc/4.0/.

\section{References}

1. National Cancer Center Japan. Cancer registration/statistics. Graph database. 2020. http://gdb.ganjoho.jp/graph_db/index ?changeLang=Submit Accessed 14 Aug 2020

2. Japan Ministry of Health, Labour, and Welfare. Overview of national medical expenses in 2017. 2019. https://www.mhlw. go.jp/toukei/saikin/hw/k-iryohi/17/index.html Accessed 14 Aug 2020 (in Japanese)

3. Ross JS, Slodkowska EA, Symmans WF, Pusztai L, Ravdin PM, Hortobagyi GN. The HER-2 receptor and breast cancer: ten years of targeted anti-HER-2 therapy and personalized medicine. Oncologist. 2009;14:320-68.

4. Japanese Breast Cancer Society. Guidelines for clinical practice of breast cancer based on scientific evidence (1) Treatment 2018 edition. Tokyo: KANEHARA \& Co., LTD; 2018. ((in Japanese)).

5. Swain SM, Baselga J, Kim SB, Ro J, Semiglazov V, Campone M, Ciruelos E, Ferrero JM, Schneeweiss A, Heeson S, Clark E, Ross G, Benyunes MC, Cortés J, CLEOPATRA Study Group. Pertuzumab, trastuzumab, and docetaxel in HER2-positive metastatic breast cancer. N Engl J Med. 2015;372:724-34.

6. Durkee BY, Qian Y, Pollom EL, King MT, Dudley SA, Shaffer JL, Chang DT, Gibbs IC, Goldhaber-Fiebert JD, Horst KC. Cost-effectiveness of pertuzumab in human epidermal growth factor receptor 2-positive metastatic breast cancer. J Clin Oncol. 2016;34(9):902-9.

7. Diaby V, Adunlin G, Ali AA, Zeichner SB, de Lima LG, Kohn CG, Montero AJ. Cost-effectiveness analysis of 1st through 3rd line sequential targeted therapy in HER2-positive metastatic breast cancer in the United States. Breast Cancer Res Treat. 2016;160(1):187-96.

8. Leung HWC, Chan ALF, Muo CH. Cost-effectiveness of pertuzumab combined with trastuzumab and docetaxel as a first-line treatment for HER-2 positive metastatic breast cancer. Expert Rev Pharmacoecon Outcomes Res. 2018;18(2):207-13.

9. Gafni A, Birch S. Incremental cost-effectiveness ratios (ICERs): the silence of the lambda. Soc Sci Med. 2006;62:2091-100.

10. Hasegawa M, Komoto S, Shiroiwa T, Fukuda T. Formal implementation of cost-effectiveness evaluations in Japan: a unique health technology assessment system. Value in Health. 2020;23(1):43-51.

11. Woods B, Sideris E, Palmer S, Latimer N, Soares M. NICE DSU technical support document 19: Partitioned survival analysis for decision modelling in health care: a critical review. 2017. http:// nicedsu.org.uk/technical-support-documents/partitioned-survivalanalysis-tsd/ Accessed 14 Aug 2020

12. Medical Economics Division, Health Insurance Bureau, Ministry of Health, Labour and Welfare (MHLW). Full Scale Introduction of Cost-Effectiveness Evaluations in Japan. Overview (2/20/2019). 2019. https://c2h.niph.go.jp/tools/system/overv iew_en.pdf Accessed 14 Aug 2020

13. Shiroiwa T, Fukuda T, Ikeda S, Takura T, Moriwaki K. Development of an Official Guideline for the Economic Evaluation of Drugs/Medical Devices in Japan. Value Health. 2017;20(3):372-8.

14. Guyot P, Ades AE, Ouwens MJ, Welton NJ. Enhanced secondary analysis of survival data: reconstructing the data from published Kaplan-Meier survival curves. BMC Med Res Methodol. 2012;1(12):9.

15. Diaby V, Ali AA, Williams KJ, Ezendu K, Soto-Perez-de-Celis E, Chavarri-Guerra Y, de Lima LG. Economic evaluation of sequencing strategies in HER2-positive metastatic breast cancer 
in Mexico: a contrast between public and private payer perspectives. Breast Cancer Res Treat. 2017;166(9):951-63.

16. Lloyd A, Nafees B, Narewska J, et al. Health state utilities for metastatic breast cancer. Br J Cancer. 2006;95:683-90.

17. Attard CL, Brown S, Alloul K, et al. Cost-effectiveness of folfirinox for first-line treatment of metastatic pancreatic cancer. Curr Oncol. 2014;21:e41-51.

18. Swain SM, Kim S, Cortés J, et al. Pertuzumab, trastuzumab, and docetaxel for HER2-positive metastatic breast cancer (CLEOPATRA study): overall survival results from a randomised, double-blind, placebo-controlled, phase 3 study. Lancet Oncol. 2013;14:461-71.
19. Ministry of Health, Labour and Welfare (MHLW). New drug list (scheduled to be listed on August 27, 2013). 2013. https:// www.mhlw.go.jp/file/05-Shingikai-12404000-Hokenkyoku-Iryou ka/0000016161.pdf Accessed 14 Aug 2020 (in Japanese)

20. Pharmaceuticals and Medical Devices Agency (PMDA). PERJETA Intravenous Infusion $420 \mathrm{mg} / 14 \mathrm{~mL}$ Examination Report (June 28, 2013). 2013. https://www.pmda.go.jp/drugs/2013/P2013 00075/450045000_22500AMX01001000_A100_1.pdf Accessed 14 Aug 2020 (in Japanese)

21. Saluja R, Cheng S, Delos Santos KA, et al. Estimating hazard ratios from published Kaplan-Meier survival curves: A methods validation study. Res Synth Methods. 2019;10(3):465-75. 